\title{
Konzepte zur Regelung der Strahlungsleistung pulsbetriebener IR-Emitter
}

\author{
Hoof, Christian; Schlottmann, Jörg; Wetzlar, Dietmar; Henning, Bernd \\ Universität Paderborn, EIM-E, Elektrische Messtechnik, Warburger Str. 100, 33098 Paderborn \\ Tel.: +49 5251 60-2294, E-Mail: hoof@emt.uni-paderborn.de
}

\section{Kurzfassung}

In optischen Messeinrichtungen (Abstandsmessung, NIR-Materialfeuchtemessung) werden häufig in einem schmalen Wellenlängenbereich strahlende, gepulste Infrarot-Emitter eingesetzt. Leistungsstarke Strahlungspulse sind notwendig, führen aber zu einer thermisch bedingten Abnahme der emittierten Strahlungsleistung und zu einer Verschiebung der Maximumwellenlänge. Die hierdurch entstehenden Messunsicherheiten werden aufgezeigt und Konzepte zu deren Verringerung dargestellt.

\section{Problemstellung}

Pulsbetriebene IR-Emitter haben vielfältige Anwendungsgebiete. Beispiele hierfür sind die optische Abstandsmessung oder die NIR-Materialfeuchtemessung. Ein großer Vorteil ist, dass bei der Erzeugung von IR-Strahlungspulsen durch Infrarot emittierende Dioden (IRED) auf mechanisch bewegte Bauteile wie z. B. Chopperräder verzichtet werden kann. Das Pulsen ermöglicht hohe Strahlungsleistungen zu erzeugen, was die Robustheit solcher Messsysteme insbesondere gegenüber Fremdstrahlungseinflüssen deutlich verbessert. Da hierbei die IR-Emitter bei großen Strömen (bis zu einigen Ampére) betrieben werden, ist aus thermischen Gründen die Begrenzung auf kurze Pulsdauern erforderlich. Durch diesen Pulsbetrieb tritt neben einer Langzeit- auch eine Kurzzeiterwärmung des Halbleitersubstrats auf, dessen Einfluss bei den jeweilen Anwendungen zu berücksichtigen ist. Die emittierte Strahlungsleistung und deren spektrale Verteilung ist von der Halbleitersubstrattemperatur abhängig. Diese wird von der Umgebungstemperatur und eine durch die Verlustleistung hervorgerufene Eigenerwärmung beeinflusst. Um reproduzierbare Messergebnisse zu erhalten, ist es notwendig, die Strahlungsleistung der IRED durch eine Regelung konstant zu halten. In einem ersten Ansatz wird die im gesamten Spektrum emittierte Strahlungsleistung auf einen konstanten Wert geregelt. Hierzu dient der Pulsstrom als Stellgröße. Alternativ zu Verwendung einer kostenintensiven Monitordiode wird ein Beobachter zur Schätzung der zu regelnden Größe vorgestellt. Eine thermisch bedingte Verschiebung der spektralen Strahlungsverteilung erfordert zu deren Vermeidung eine thermoelektrische Kühlung, die in technischer Hinsicht oft nicht infrage kommt oder - wie hier vorgestellt - zur Korrektur dieses Einflusses eine Berücksichtigung mithilfe eines mathematischen Modells.

\section{IRED-Strahlungsmodell}

Die relative spektrale Strahlungsleistung $S_{\lambda}$ ist proportional zum Produkt aus Zustandsdichte und Bolzmannverteilung der freien Ladungsträger [SCH06]. Mit Hilfe dieses Zusammenhangs lässt sich die Maximumwellenlänge $\lambda_{p}$ bestimmen. Zu beachten ist, dass der Bandabstand für InGaAs-IRED temperaturabhängig ist und von dem Materialparameter $x\left(\mathrm{Ga}_{x} \mid \mathrm{n}_{1-x} \mathrm{As}\right)$ abhängt [PRB91]. Hieraus lässt sich die temperaturbedingte Verschiebung von $\lambda_{p}$ erklären.

$$
\begin{gathered}
S_{\lambda}(\lambda, T, x): \sqrt{\frac{h c}{\lambda}-E_{g}(T, x)} \cdot e^{\left(-\frac{h c}{\lambda \cdot k_{B} T}\right)} \\
\lambda_{\mathrm{p}}(T, x)=\frac{h \cdot c}{E_{g}(T, x)+\frac{1}{2} k T}
\end{gathered}
$$

Die Abbildung 1 zeigt beispielhaft einen Verlauf der auf den Maximalwert normierten relativen spektralen Strahlungsleistung. 


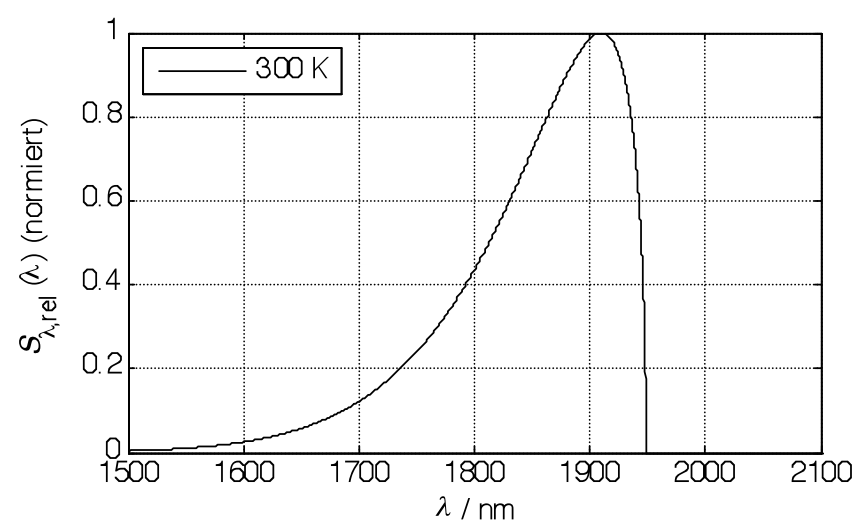

\section{Abbildung 1: Theoretische erwartete relative spektrale Strahlungsleistung normiert auf den Maximalwert}

\section{Messung des Emissionsspektrums einer IRED}

Zur Verifikation dieses theoretischen Zusammenhangs wird im Folgenden das Emissionsspektrum einer IRED des Typs LED19PRW mit Hilfe eines Monochromators bestimmt. Dabei ermöglicht die Variation der Gitterstellung des Monochromators wellenlängenselektive Messungen der Strahlungsleistung. Die Messungen werden bei verschiedenen Umgebungstemperaturen ausgeführt, um die Temperaturabhängigkeit der spektralen Emission der IRED zu bestimmen. Abbildung 2 zeigt den Messaufbau. Die IRED wird mit einem pulsförmigen Strom $i_{F}$ betrieben und die emittierte Strahlung mit Hilfe eines Monochromators analysiert, der in einem Wellenlängenbereich von 1,6 $\mu \mathrm{m}$ bis $2 \mu \mathrm{m}$ und einer spektralen Breite von $\Delta \lambda$ um die Mittenwellenlänge $\lambda_{M}$ durchgestimmt wird. Pulsdauer und Pulspause sind dabei so zu wählen, dass Umgebungstemperatur und Substrattemperatur der IRED als gleich angenommen werden können.

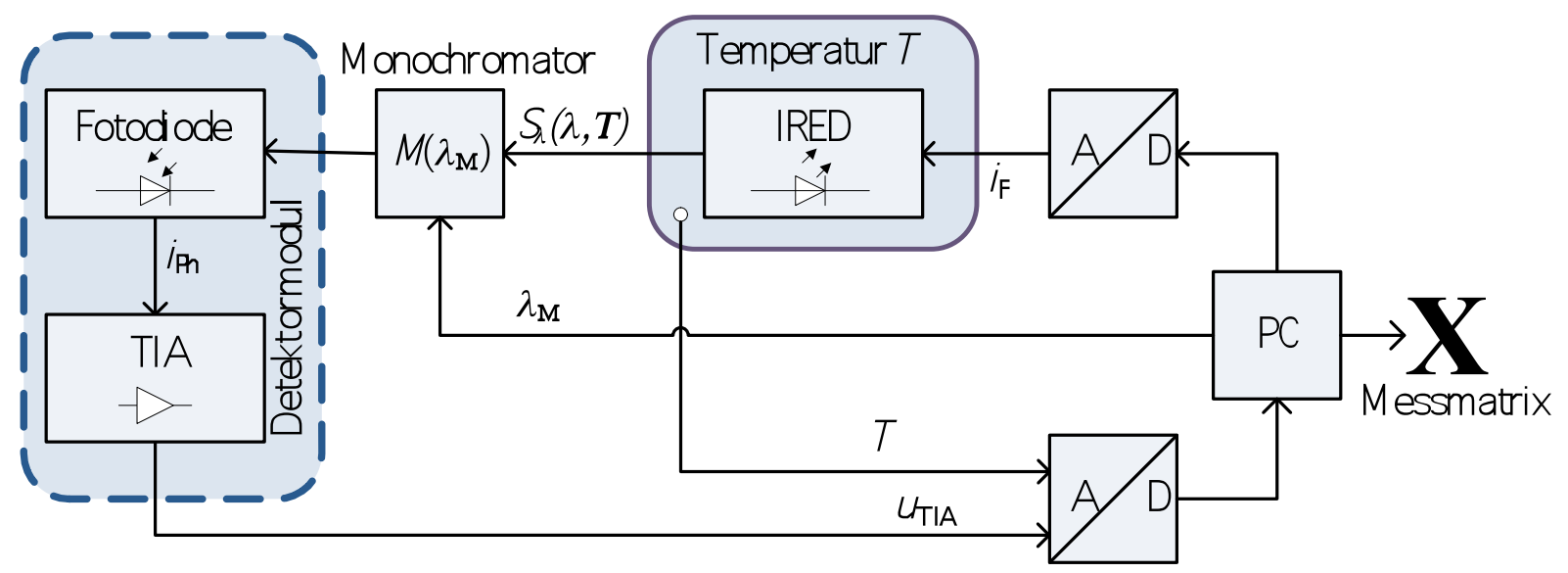

\section{Abbildung 2: Schematischer Messaufbau zur Bestimmung des Emissionsspektrums einer IRED mit einem Monochromator}

Zur Messung der Strahlungsleistung wird eine IR-Fotodiode eingesetzt, dessen Fotostrom $i_{\text {ph }}$ proportional zur Bestrahlungsstärke ist. Dieser Fotostrom wird mit einem Detektor der spektralen Empfindlichkeit $s_{\lambda}$ und einem Transimpedanzverstärker (TIA) in eine Spannung $u_{\text {TIA }}$ überführt. Die relative spektrale Transmission des Monochromators $M(\lambda)$ wirkt wie ein schmalbandiges Bandpassfilter der Breite $\Delta \lambda$. Für die am Detektormodul gemessene Spannung gilt:

$$
u_{\mathrm{TIA}}\left(\lambda_{\mathrm{M}}, T\right): \int_{0}^{\infty} M\left(\lambda_{{ }_{\mathrm{M}}} \lambda_{\mathrm{M}}\right) S_{\lambda}\left(\lambda_{\mathrm{M}} T\right) s(\lambda) d \lambda \approx S_{\lambda}(\lambda, T) s(\lambda) \cdot \Delta \lambda
$$

Abbildung 3 zeigt die bei einer Umgebungstemperatur von $30,6{ }^{\circ} \mathrm{C}$ aufgenommenen Pulsantworten für unterschiedliche Wellenlängen im Bereich von $1,6 \mu \mathrm{m}$ bis $2,1 \mu \mathrm{m}$. 


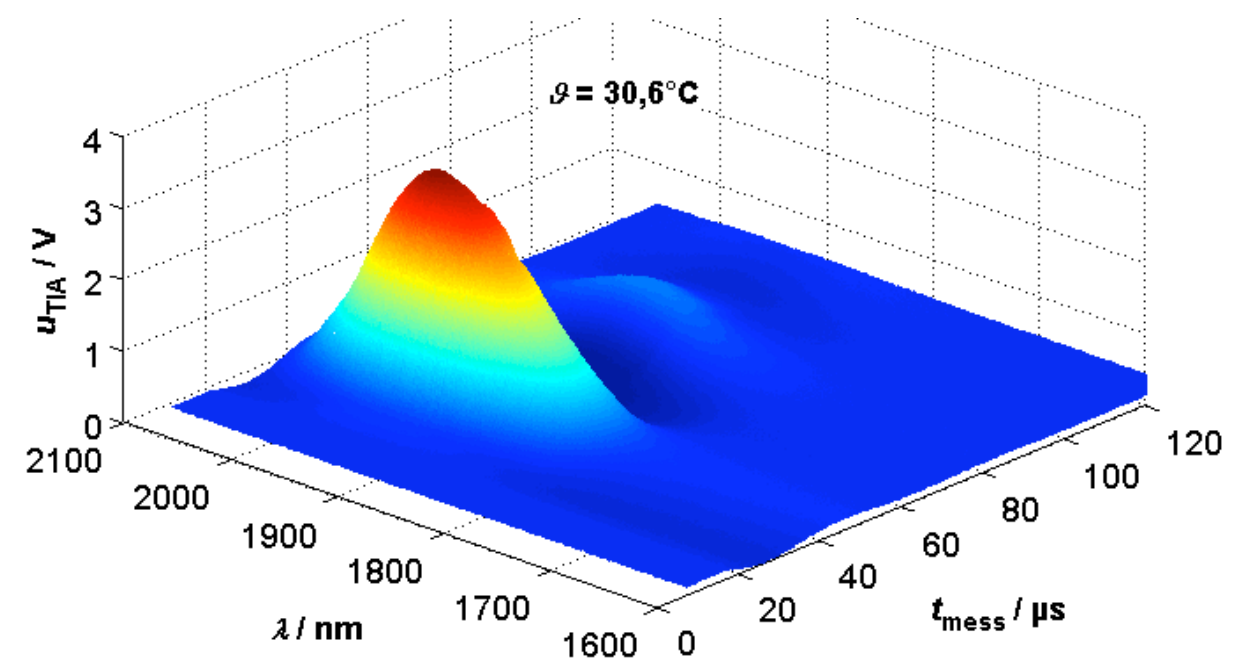

\section{Abbildung 3: Bildung der Messmatrix $X$ aus Pulsantworten abhängig von der Monochromatorwellenlänge, $30,6^{\circ} \mathrm{C}$}

Die aufgenommenen Pulsantworten am Detektormodulausgang sind beinflusst von stochastischen und dynamischen Störungen. Aus diesen Daten lässt sich das Emissionsspektrum mit dem Verfahren der Hauptkomponentenanalyse (Principal Component Analysis, PCA) extrahieren. Mit Hilfe der PCA können aus großen Datenmengen Merkmale extrahiert und somit Daten reduziert werden. In diesem Fall wird die Messmatrix $\mathbf{X}$ wie folgt aufgestellt. Für $n$ Monochromatorgitterstellungen wird das Pulssignal am Detektormodulausgang $m$ mal abgetastet. Abbildung 4 veranschaulicht das Vorgehen. Jeder Zeitverlauf repräsentiert eine andere Wellenlänge.

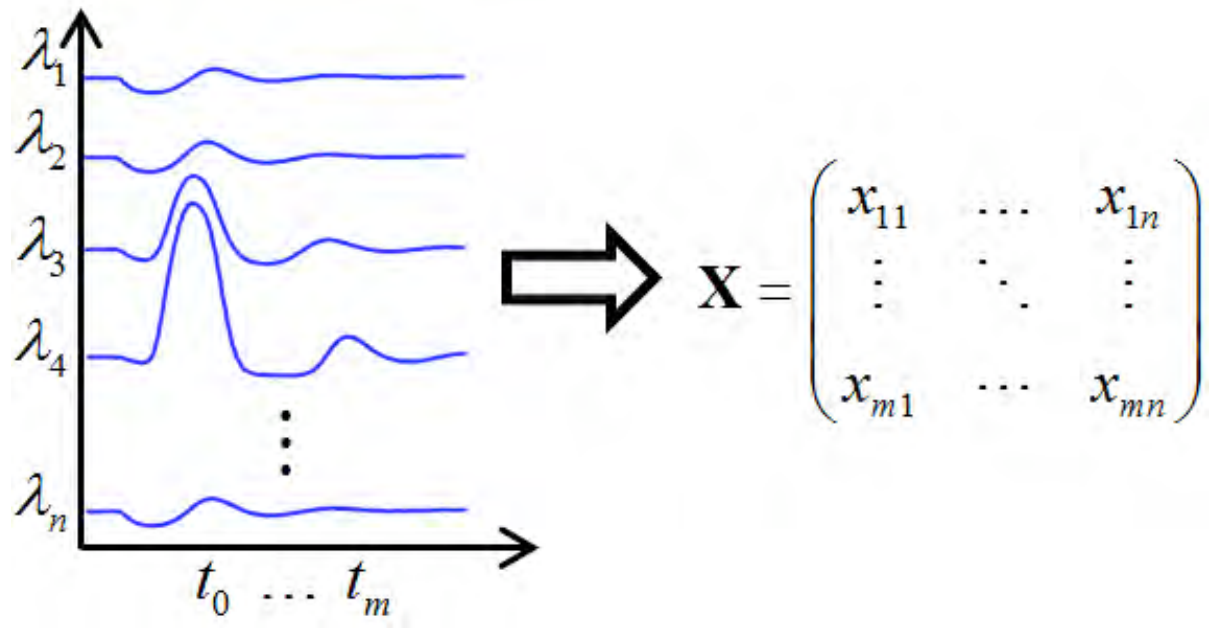

Abbildung 4: Bestimmung der Messmatrix X

Die Messmatrix $\mathbf{X}$ besitzt somit die Dimension $(m \times n)$, wobei $m$ die Anzahl der Beobachtungen (zeitlicher Verlauf) bei $n$ Wellenlängen repräsentiert. Die Hauptkomponente $\mathbf{t}_{i}$ erhält man durch Transformation der Messmatrix $\mathbf{X}$ mit dem Eigenvektor $\mathbf{p}_{i}$ der Kovarianzmatrix von $\mathbf{X}$.

$$
\mathbf{t}_{i}=\mathbf{X p}_{i} \quad i=1 \mathrm{~K} n
$$

Die erste Hauptkomponente $\mathbf{t}_{1}$ liefert die gesuchte markante Information, die proportional zur relativen spektralen Strahlungsleistung $S_{\lambda}$ ist. Die Hinzunahme weiterer Hauptkomponenten verbessert das Ergebnis unwesentlich, da im ersten Eigenwert ca. 95\% der Gesamtvarianz enthalten ist. Die PCA wird dabei für die Messreihen bei verschiedenen Temperaturen durchgeführt. Die jeweils erste Hauptkomponente liefert folgendes Ergebnis (Abbildung 5): 


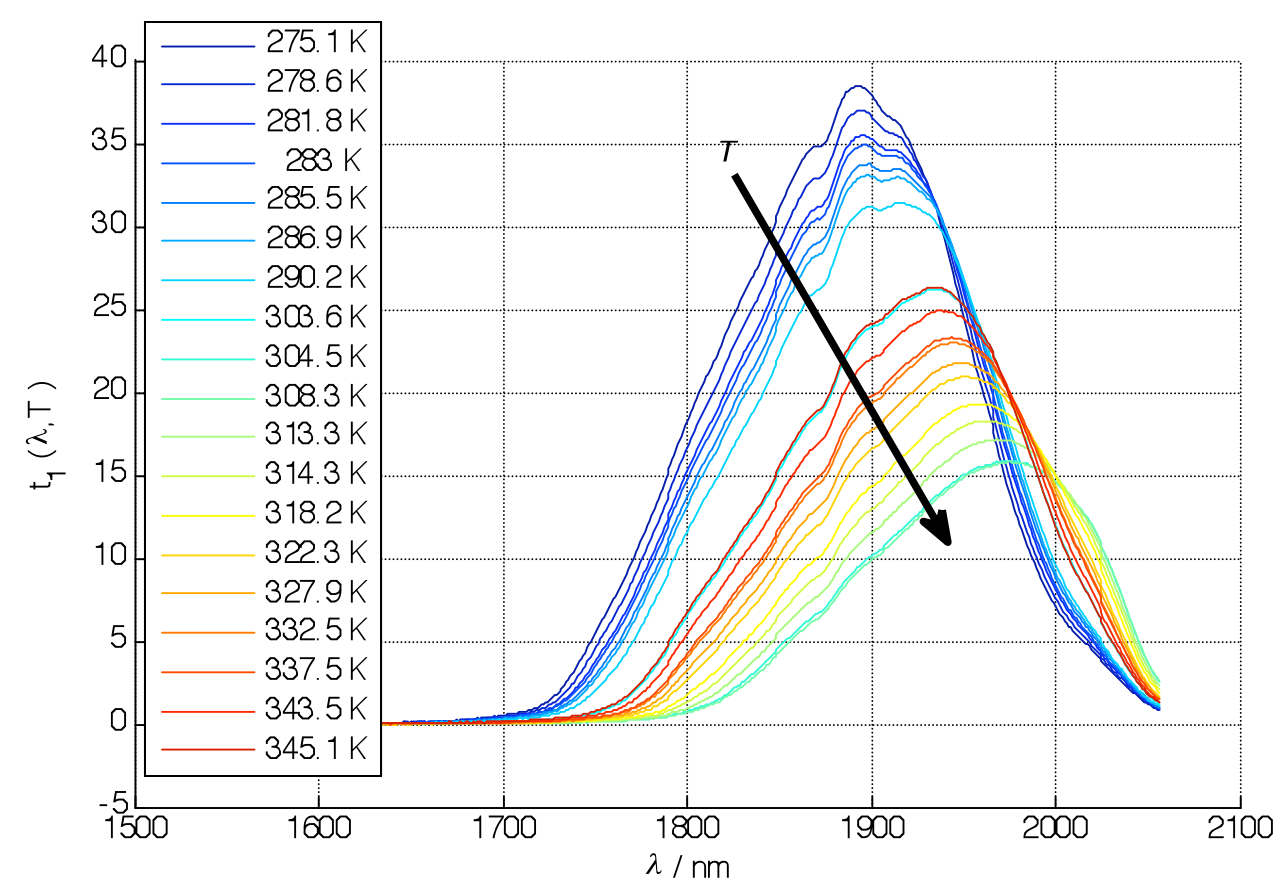

\section{Abbildung 5: Gemessene spektrale emittierte Leistung einer IRED LED19PRW für verschiedene Temperaturen}

$\mathrm{Zu}$ beachten ist, dass auf der Ordinate nicht die Detektormodulausgangsspannung, sondern eine dimensionslose Zahl proportional zur relativen spektralen Strahlungsleistung $S_{\lambda}$ steht. Mit steigender Temperatur nimmt bei gleichem Pulsstrom die emittierte Strahlungsleistung ab, $d$. $h$. die Fläche unter den Kurven verringert sich und die Maximumwellenlänge verschiebt sich zu größeren Wellenlängen.

\section{Phänomenologisches Modell}

Das oben beschriebene theoretische Modell beschreibt zwar die temperaturbedingte Verschiebung der Maximumwellenlänge, kann jedoch nicht die temperaturabhängige Abnahme der gesamten emittierten Strahlungsleistung erklären. Auch die im Modell auftretende Grenzwellenlänge (Abbildung 1), bedingt durch einen minimalen Bandabstand, kann messtechnisch nicht bestätigt werden. Die Messung ergibt einen asymmetrischen, glockenförmigen Verlauf. Die mathematische Modellierung dieses messtechnischen Befundes (Abbildung 5) lässt sich durch Überlagerung zweier Gauß-Verteilungen darstellen.

$$
t_{1}(\lambda, T): S_{\lambda, \text { Modell }}(\lambda, T)=a_{1} \cdot e^{-\left(\left(\lambda-\left(b_{1} \cdot T+b_{2}\right)+b_{0}\right) c_{1}\right)^{2}}+a_{21} e^{\left(a_{22} T\right)} \cdot e^{-\left(\left(\lambda-\left(b_{1} \cdot T+b_{2}\right)\right)\left(c_{21} T+c_{22}\right)\right)^{2}}
$$

Das Ergebnis dieser Approximation zeigt die Abbildung 6. Die Fläche repräsentiert das Modell durch Überlagerung zweier Gauß-Verteilungen, die Punkte sind Messwerte. 


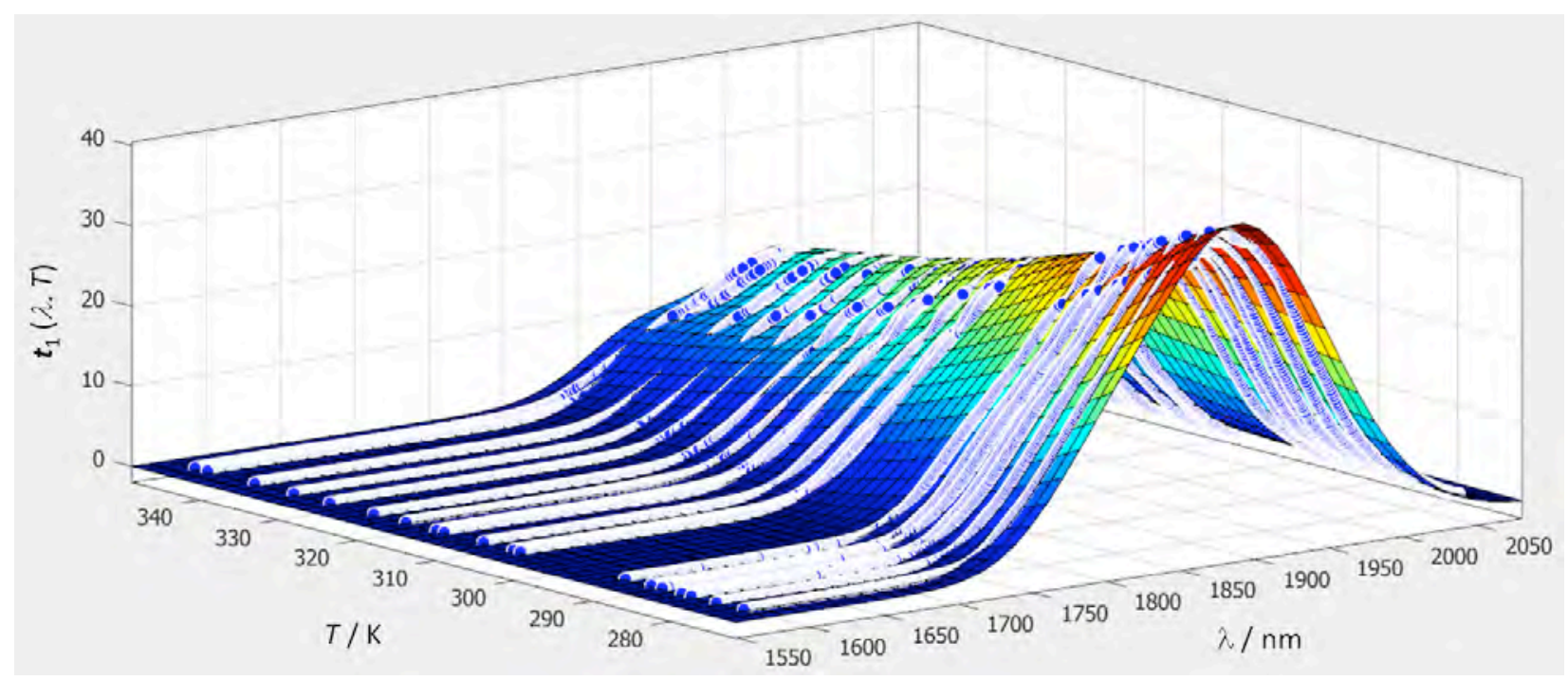

Abbildung 6: Annäherung durch zwei Gauß-Kurven mit durch PCA interpretierten Messergebnissen

\section{Anwendung: Optische Abstandsmessung}

Für eine optische Abstandsmessung in Umgebungen mit großer Fremdstrahlung lassen sich nur gepulste IRED einsetzen, bei denen die über den gesamten Wellenlängenbereich emittierte Strahlungsleistung $\Phi_{\mathrm{e}}$ konstant sein muss. Dies erfordert eine Regelung. Insbesondere im IR-Bereich oberhalb von $1 \mu \mathrm{m}$ muss oft aus Kostengründen auf eine Monitordiode verzichtet werden. Alternativ kann die zu regelnde Größe aber auch durch einen Beobachter aus messbaren elektrischen IRED-Größen geschätzt werden (Abbildung 7). Das Konzept sieht vor, dass bei kurzen Pulsdauern anstelle der Strahlungsleistung die Energie eines Pulses zur Regelung genutzt wird. Durch dieses Vorgehen lässt sich der notwendige Hardwareaufwand zur Strahlungsleistungsregelung deutlich reduzieren.

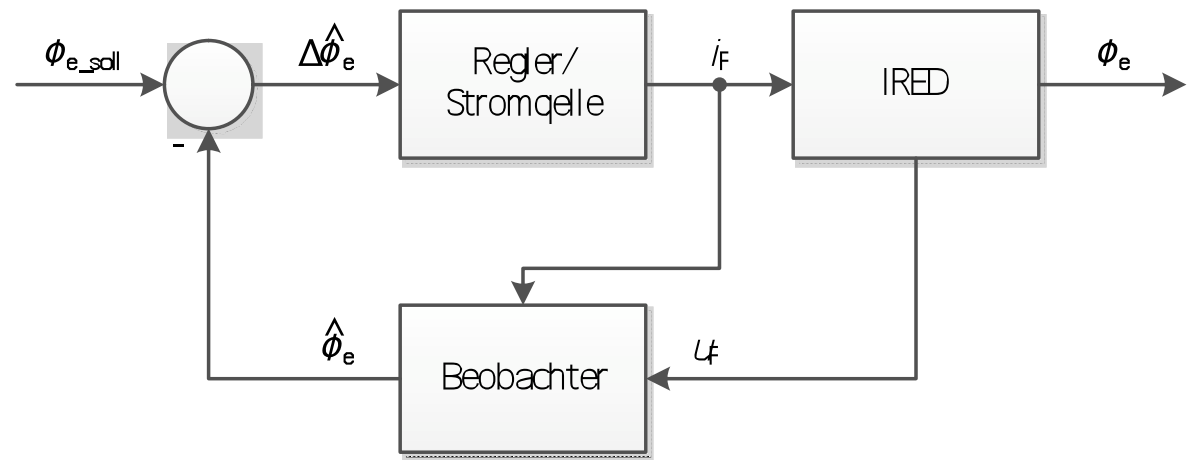

Abbildung 7: Regelkreis mit Beobachter

Um die Abnahme der Strahlungsleistung durch die Kurzzeiterwärmung während eines Pulses vernachlässigen zu können muss die Pulsdauer möglichst kurz gewählt werden.

$$
\hat{Q_{e}}=t \int_{0}^{T_{P}} d t_{\mathrm{e}}\left(\hat{\phi} T \approx{ }_{\mathrm{e} P}^{-}\right.
$$

Die Strahlungsenergie $Q_{\mathrm{e}}$ pro Puls der Dauer $T_{\mathrm{P}}$ ist proportional zur mittleren Strahlungsleistung $\widehat{O}_{\mathrm{e}}$. 


\section{Beobachterentwurf}

Der Beobachter soll bei unterschiedlichen Temperaturen den Wert der Strahlungsleistung $\Phi_{\mathrm{e}}$ einer IRED vorhersagen. Dazu werden die elektrischen Größen genutzt, die Vorwärtsspannung $u_{F}$ über der IRED und der Strom $i_{F}$, mit dem der Emitter gepulst wird. Die Ausgangsspannung $u_{\text {TIA }}$ des Detektormoduls ist proportional zum Produkt aus spektraler Empfindlichkeit des Detektors $s_{\lambda}$ und der spektralen Strahlungsleistung $S_{\lambda}$ des Emitters.

$$
\left.u \hat{Q}_{\mathrm{IIA}} \sim \dot{k} \mathrm{Ph}_{\mathrm{S}} \sim{ }_{\lambda \mathrm{e}} \sim \int_{0}^{\infty} d \lambda\right)(\lambda) \lambda
$$

Das Blockschaltbild Abbildung 8 zeigt den zum Beobachterentwurf genutzten Versuchsaufbau.

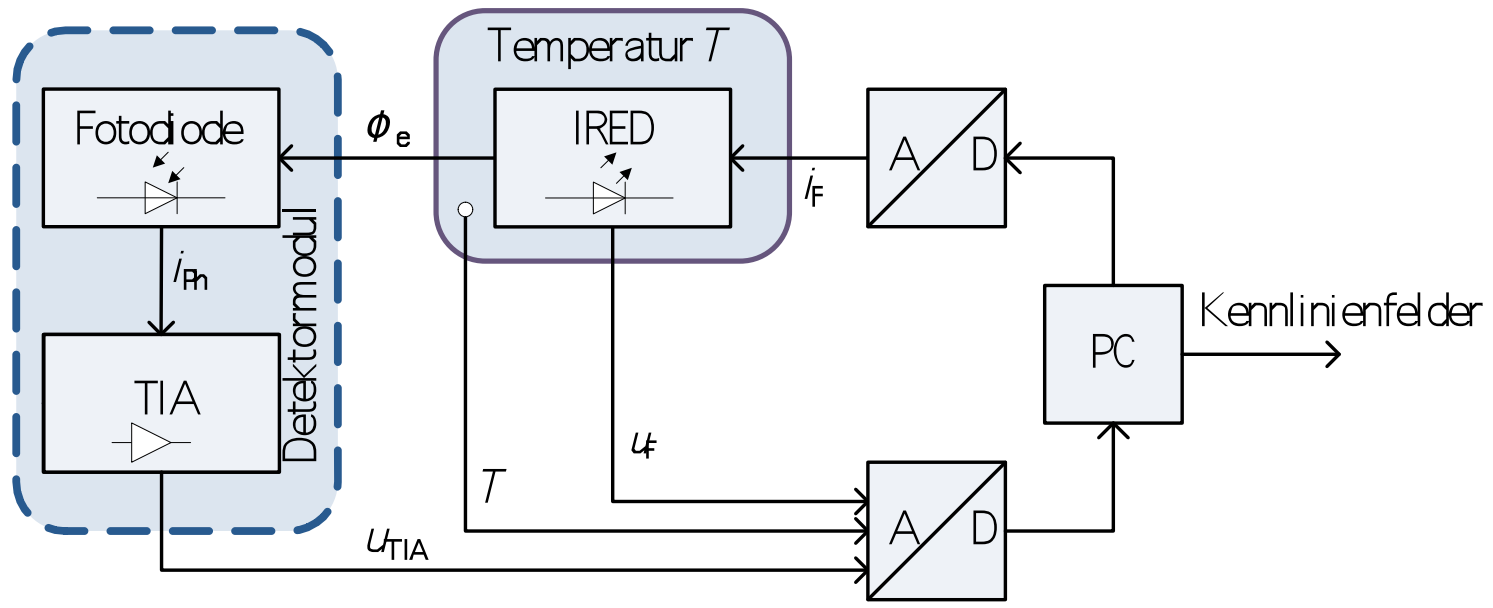

\section{Abbildung 8: Versuchsaufbau - Kennlinienfeldbestimmung}

Die gemessene elektrische Leistung einer IRED ist über die charakteristische Strahlungsausbeute $\eta_{\mathrm{e}}$ mit der emittierten Strahlungsleistung verknüpft. Die Größe $\eta_{\mathrm{e}}$ ist der Quotient aus $\Phi_{\mathrm{e}}$ und der elektrischen Leistung $P_{\mathrm{el}}$, welche sich aus der Vorwärtsspannung $u_{\mathrm{F}}$ und dem Strom $i_{\mathrm{F}}$ des Emitters ergibt.

$$
\eta_{\mathrm{e}}=\frac{\hat{O}_{\mathrm{e}}}{P_{\mathrm{el}}}=\frac{\hat{O}_{\mathrm{e}}\left(i_{\mathrm{F}}, T_{\mathrm{j}}\right)}{u_{\mathrm{F}}\left(i_{\mathrm{F}}, T_{\mathrm{j}}\right) \cdot i_{\mathrm{F}}}=\eta_{\mathrm{e}}\left(i_{\mathrm{F}}, T_{\mathrm{j}}\right)
$$

Die Abbildung 9 zeigt die Kennlinienfelder zur Bestimmung der Strahlungsausbeute. Die Grafiken verdeutlichen die Abhängigkeiten der relevanten Größen $\Phi_{\mathrm{e}}$ und $u_{\mathrm{F}}$ zur Bestimmung der Strahlungsausbeute $\eta_{\mathrm{e}}\left(i_{\mathrm{F}}, T_{\mathrm{j}}\right)$ von der Substrattemperatur $T_{\mathrm{j}}$ (hier dargestellt als $\vartheta_{\mathrm{j}}$ in ${ }^{\circ} \mathrm{C}$ ) und dem Strom $i_{\mathrm{F}}$.
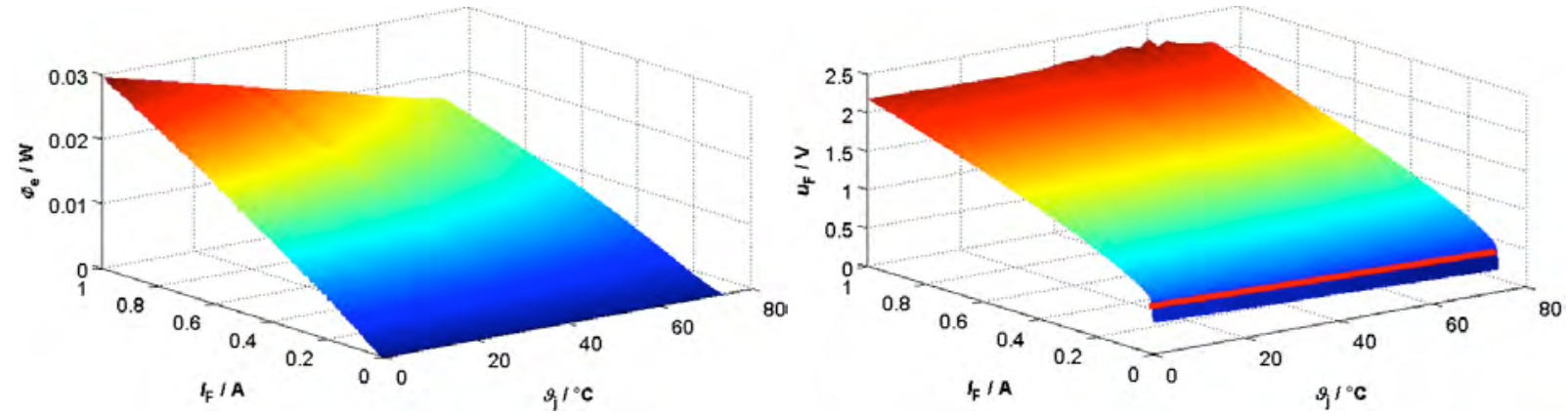

Abbildung 9: Kennlinienfelder einer IRED LED19PRW, links: Strahlungsleistung $\Phi_{\mathrm{e}}$, rechts: Vorwärtsspannung $u_{\mathrm{F}}$

Aus den in den Kennlinienfeldern gezeigten Daten wird mit Hilfe eines Polynomansatzes ein mathematischer Ausdruck für die Strahlungsausbeute $\eta_{\mathrm{e}}$ bestimmt, welche den Zusammenhang zwischen 
der Strahlungsleistung, der Vorwärtsspannung und dem Strom durch die IRED bei unterschiedlichen Temperaturen herstellt. Die Substratstemperatur $T_{\mathrm{j}}$ wird durch Messung der IRED-Vorwärtsspannung $u_{\mathrm{F}}$ bei kleinen Strömen (rote Linie in Abbildung 9, rechts) über ein Diodenmodell bestimmt. Somit lässt sich aus $u_{\mathrm{F}}$ und $i_{\mathrm{F}}$ die Strahlungsleistung schätzen.

Die elektrische Verlustleistung $P_{\mathrm{v}}$, welche nicht in Strahlungsleistung gewandelt wird, trägt zu Eigenerwärmung der IRED bei. Dabei gilt:

$$
P_{\mathrm{v}}=\left(1-\eta_{\mathrm{e}}\right) P_{\mathrm{el}}
$$

\section{Ergebnisse zur Strahlungsleistungsregelung}

Abbildung 10 zeigt die Wirkung der Regelung der gemittteten Strahlungsleistung anhand eines Aufheizvorganges. Eine IRED in einer Messkammer wird für ca. 6 Minuten mit einem Heißluftgebläse von etwa $23^{\circ} \mathrm{C}$ auf etwa $50{ }^{\circ} \mathrm{C}$ erwärmt. Ohne Regelung sinkt die emittierte Strahlungsleistung auf etwa $70 \%$ ihres Ausgangswertes ab. Mit Regelung vermindert sich die Strahlungsleistung nur noch um weniger als $5 \%$.
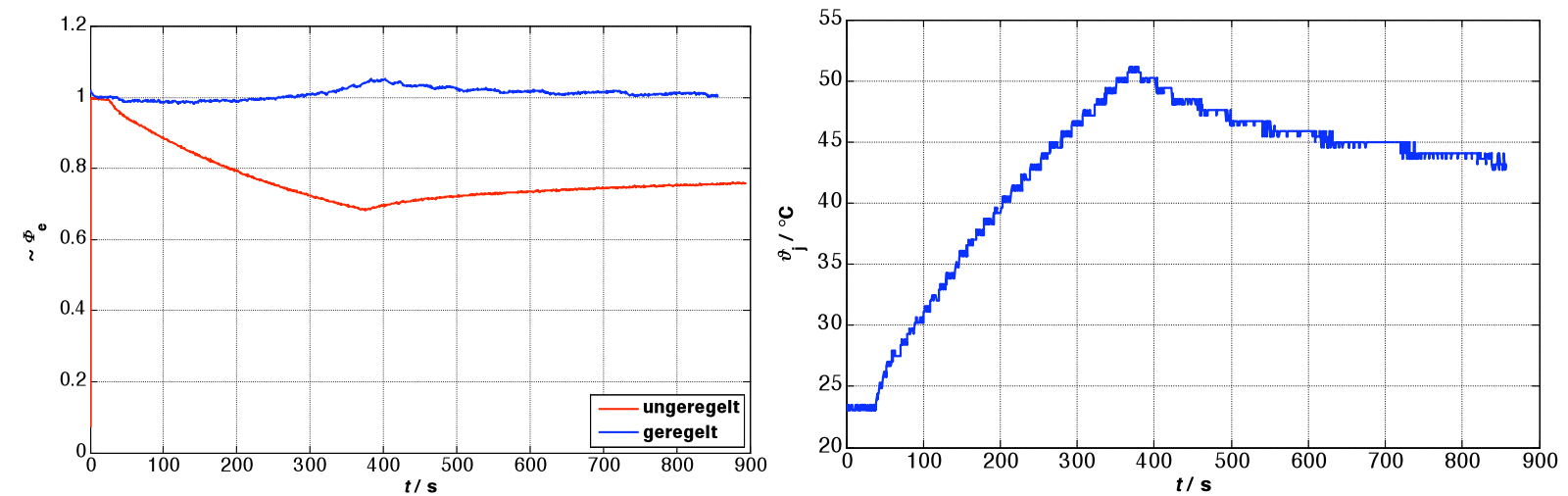

Abbildung 10: Mit einem InGaAs-Detektor gemessene Strahlungsleistung einer LED19PRW normiert auf den Anfangswert, $i_{\mathrm{F}}$ konstant Links: ungeregelter (rot) und geregelter (blau) Betrieb Rechts: Zeitlicher Verlauf der Substrattemperatur (ermittelt aus $u_{\mathrm{F}}$ )

Die Wirkung der Regelung bei abrupter Abkühlung der IRED durch ein Kühlmittel (Kältespray) ist in Abbildung 11 dargestellt.

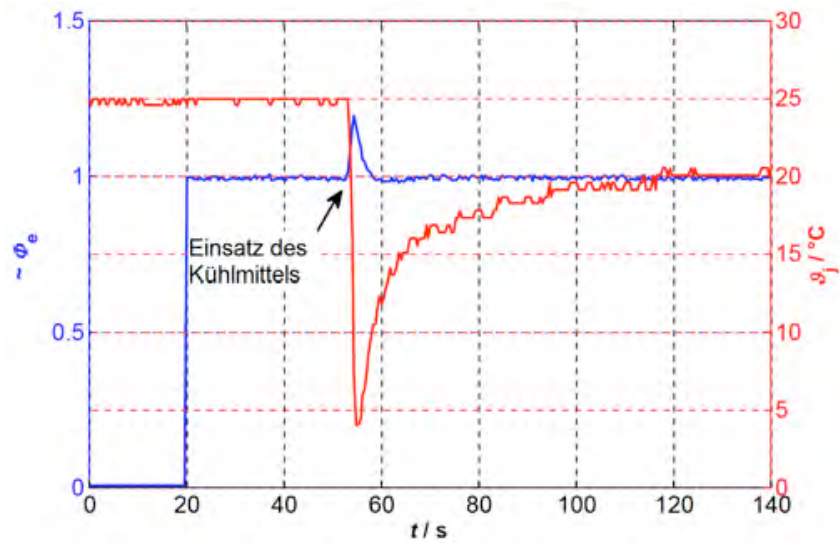

\section{Abbildung 11: Strahlungsleistungsregelung bei Abkühlung mit Kühlmittel Blau: Zeitlicher Verlauf der Strahlungsleistung Rot: Zeitlicher Verlauf der Substrattemperatur (ermittelt aus $u_{F}$ )}




\section{Anwendung: NIR-Materialfeuchtemessung}

Zur berührungslosen Materialfeuchtebestimmung wird u. a. das Infrarotreflexionsverfahren (NIR) eingesetzt. Um das Messobjekt zu bestrahlen, lassen sich in unterschiedlichen Wellenlängenbereichen strahlende IR-Emitter nutzen. Wie in Abbildung 12 dargestellt, ist über die Auswertung des vom Messobjekt diffus reflektierten Strahlungsanteils ein Rückschluss auf die Materialfeuchte möglich [WET98][WET03][WK09].

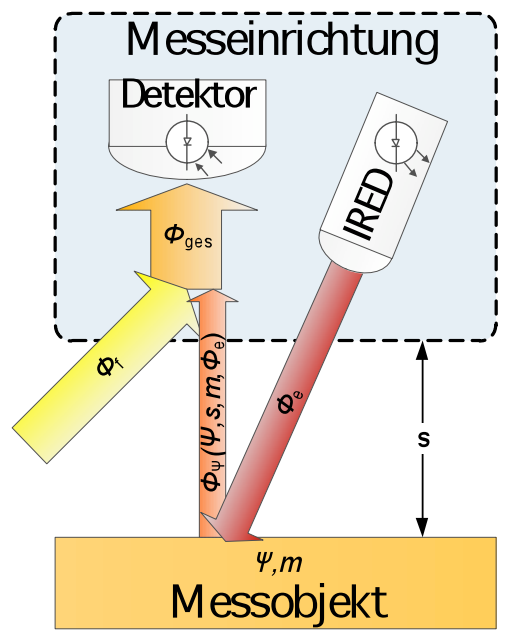

\section{Abbildung 12: Auftretende Strahlungsgrößen in einem Materialfeuchtemesssystem}

Die detektierbare Strahlungsleistung $\Phi_{\text {ges }}$ setzt sich aus einem Fremdstrahlungsanteil $\Phi_{\mathrm{f}}$ und dem Nutzstrahlungsanteil $\Phi_{\Psi}$ zusammen. Zur Erhöhung der Strahlungsleistung werden die IRED gepulst. Eine Regelung der Strahlungsleistung nach Abbildung 7 führt zu einer konstanten emittierten Strahlungsenergie je Puls. Eine wellenlängenabhängige Verschiebung der Maximumwellenlänge wird hier jedoch nicht berücksichtigt. Im Folgenden wird gezeigt, dass hierdurch Fehler bei einer Materialfeuchtebestimmung entstehen.

Das Messprinzip eines NIR-Materialfeuchtemesssystems beruht auf der Erfassung der vom feuchten Messobjekt diffus reflektierten Strahlung. Der Messeffekt wird durch eine Wechselwirkung zwischen elektromagnetischer Welle und den Wassermolekülen verursacht. Es handelt sich dabei um die Anregung von Resonanzschwingungen, so zeigt die Absorption eine charakteristische Abhängigkeit von der Wellenlänge. Abbildung 13 zeigt den wellenlängenabhängigen Absorptionskoeffizienten für flüssiges Wasser. Bei ca. 1,9 $\mu \mathrm{m}$ ist die Absorption besonders stark ausgeprägt. Eine weitere Absorptionsbande liegt bei etwa $1,4 \mu \mathrm{m}$.

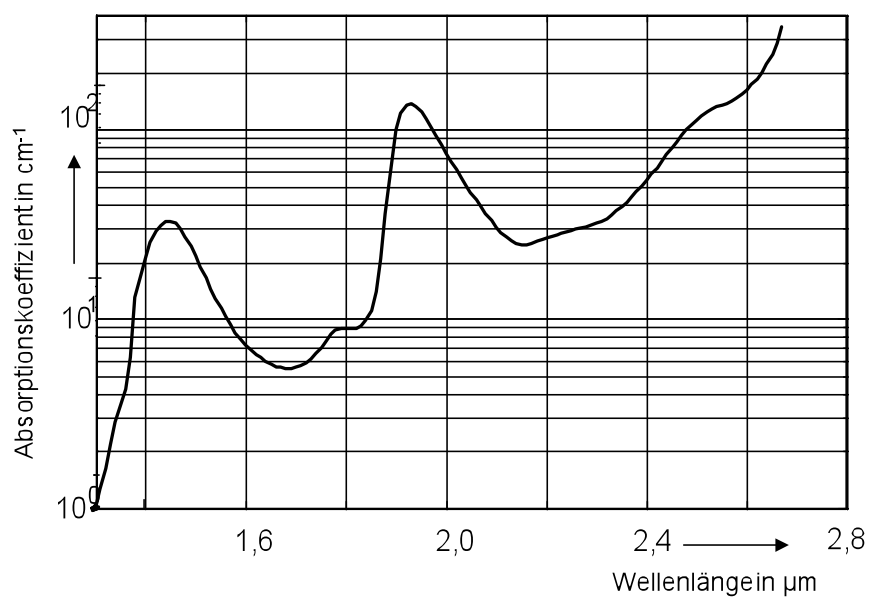

Abbildung 13: Wellenlängenabhängiger Verlauf des Absorptionskoeffizienten [WET98] 
In Abhängigkeit von der Substrattemperatur der IRED verschiebt sich die Maximumwellenlänge der IRED gegenüber der Wasserbande. Infolge dessen ist der Messwert einer Feuchtebestimmung temperaturabhängig. Um diesen Einfluss bei der Auswertung der Messsignale berücksichtigen zu können, kann das zuvor entwickelte mathematische Modell der IRED nach Abbildung 6 eingesetzt werden.

Die Abbildung 14 zeigt den Einfluss der Wasserabsorptionsbande bei 1,9 $\mu \mathrm{m}$ auf die temperaturabhängige relative spektrale Strahlungsleistung $S_{\lambda}(\lambda, T)$. Allerdings iszt es mit Hilfe einer Fotodiode nur möglich, eine zur Strahlungsleistung $\Phi_{\mathrm{e}}$ proportionale integrale Größe zu erfassen. Sowohl Temperatur, als auch die Materialfeuchte beeinflussen das Messsignal - beide Größen sind verkoppelt. Temperaturzunahme und Erhöhung der Materialfeuchte führen zu einer Abnahme des Messsignals bei Messung im Bereich einer Wasserabsorptionsbande.
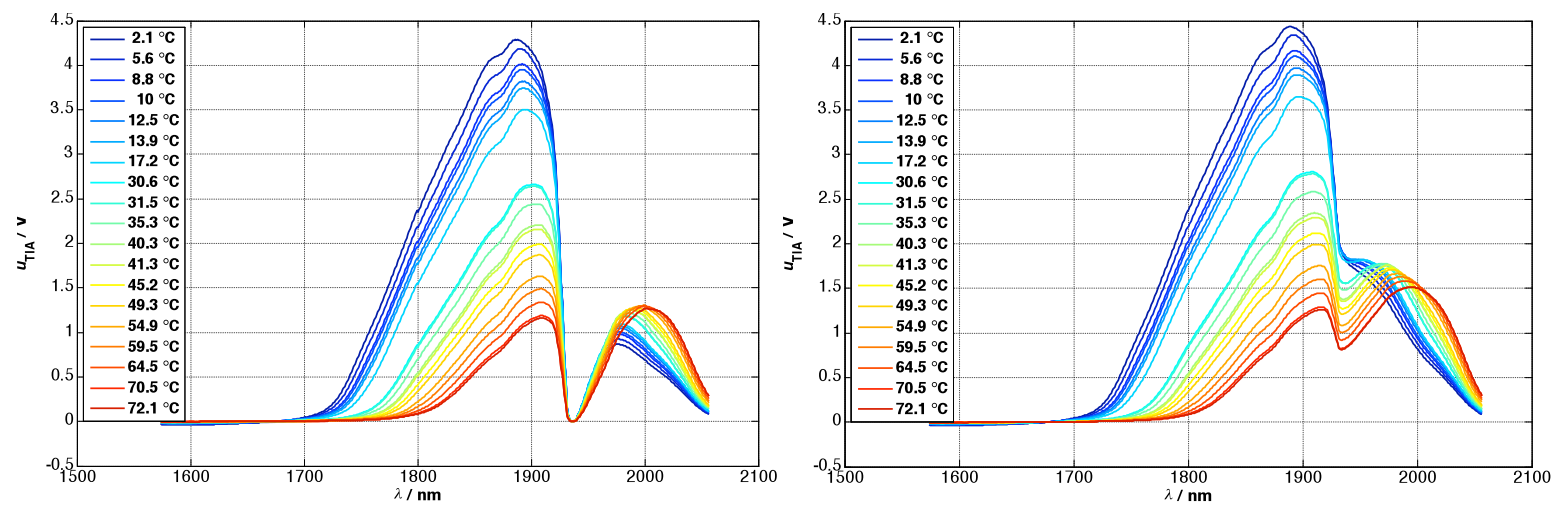

\section{Abbildung 14: Relative spektrale Strahlungsleistung einer IRED LED19PRW bei der 1,9 $\mu \mathrm{m}$-Wasserbande bei verschiedenen Substrattemperaturen. Links: starke Abschwächung, rechts: geringere Abschwächung}

Ein möglicher Ansatz, die Einflüsse der Materialfeuchte $\Psi$ von den Einflüssen der temperaturbedingten Verschiebung der Maximumwellenlänge zu entkoppeln basiert auf einem Verfahren der Ausgleichsrechnung vermittelnder Beobachter [ZUR65] und ist vergleichbar anzuwenden an den in [WK09] beschriebenen Algorithmus zur Entkopplung der beiden Größen Materialfeuchte $\Psi$ und Messgutabstand $s$.

Ein alternativer Ansatz besteht darin, den Temperatureinfluss in $S_{\lambda, \text { RED-Typ,Modell }}(\lambda, T)$ für jede der verwendeten IRED gemäß dem Modell nach Abbildung 6 zu berücksichtigen. Hierzu ist zunächst durch Simulation die Abhängigkeit der Detektorausgangsspannung von der Materialfeuchte und von der Temperatur darzustellen und daraus ein funktionaler Zusammenhang $u_{T I A, I R E D-T y p}(\Psi, T)$ zu entwickeln. Die Verwendung in unterschiedlichen Wellenlängenbereichen strahlender IRED führt zu einem Gleichungssystem. Dieses aufgelöst liefert die gesuchte Materialfeuchte.

\section{Zusammenfassung}

Der Pulsbetrieb von IRED mit großen Stromstärken führt zu thermisch bedingter Abnahme der emittierten Strahlungsleistung und zur Verschiebung der Maximumswellenlänge. Beispielsweise bei der optischen Abstandsmessung ist es ausreichend, die gesamte emittierte Strahlungsleistung durch eine Regelung konstant zu halten. Um keine Monitordiode einsetzen zu müssen, wurde hier ein Beobachteransatz vorgestellt. Im Falle der NIR-Materialfeuchtemessung ist zusätzlich die Verschiebung der Maximumswellenlänge zu berücksichtigen. Dieser Effekt wurde messtechnisch verifiziert und mit einem mathematischen Modell beschrieben. Basierend hierauf wurden zwei alternative Ansätze zu deren Kompensation skizziert. 
Literatur

[PRB91]

[SCH06] Schubert, E. Fred: Light-emitting diodes. 2nd. Edition Cambridge, New York: Cambridge University Press, 2006

[WET98] Wetzlar, D.: Prozessbegleitende Materialfeuchtemessung nach dem NIR-Prinzip bei schüttfähigen Gütern, Hydromechanisation 10 - Tenth International Conference on Hydromechanisation, Zakopane, Polen, 4.-7. Mai 1998. In: Proceedings S. 221-227

[WET03] Wetzlar, D.: NIR Moisture Measurement with Selectively Radiating Sources, Sensor 2003, Int. Conf. for Sensors, Nuremberg, Germany May 13-15, 2003

[WK09] Wetzlar, D.; Krumme, M.: Berührungslose Materialfeuchtemessung nach dem NIRPrinzip bei schnell variierendem Messgutabstand. 4. CMM-Workshop Innovative Feuchtemessung in Forschung und Praxis, Bad Herrenalb, 30.09. - 01.10.2009, Berichtsband, S.101-111

[ZUR65] Zurmühl, R.: Praktische Mathematik für Ingenieure und Physiker, 5. Auflage, Springer Berlin, 1965 